\title{
$\omega$-3 Polyunsaturated Fatty Acids on the Prognosis of Multiple Sclerosis: The Effect of Eicosapentaenoic acid
}

\author{
Serafina Salvati* and Antonella Di Biase
}

Department Veterinary Public Health \& Food Safety-IstitutoSuperiore di Sanità-Rome, Italy

\begin{abstract}
The effect of nutrition and dietary supplements on the course of multiple sclerosis is a topic of great interest to both patients and clinicians. The influence of $\omega-3$ polyunsaturated fatty acids (PUFAs) on the development and course of multiple sclerosis (MS) has been under investigation. A number of small, uncontrolled clinical trials with fish oil (rich in eicosapentaenoic acid (EPA; 20:5 $\omega$-3) and docosahexaenoic acid (DHA; 22:6 $\omega-3$ ) have indicated a possible beneficial effects of $\omega-3$ PUFAs, but controlled trials have not been able to draw definitive conclusions. Among $\omega$-3 PUFAs, EPA has the most potent effect on myelinogenesis and inflammatory process, thus the conflicting results could be due to the different mixtures of EPA and DHA contained in the various sources of fish oil. This review discusses on the possible role of EPA in demyelinating diseases.
\end{abstract}

Keywords: $\omega-3$ polyunsaturated fatty acids; Eicosapentaenoic acid; Docosahexaenoic acid; Multiple sclerosis; Fish oil

\section{Introduction}

Multiple sclerosis (MS) is a chronic neurological disease that is a major cause of disability in young adults in Europe and North America [1]. The clinical course of MS is unpredictable and varies individually, with some patients experiencing minimal impairment. The majority of patients have a form of MS referred to as relapsing-remitting MS (RRMS) characterized by periods of clinical stability that are interrupted by relapses. MS is generally considered an immune-mediated disease characterized by disseminated foci of inflammatory demyelination affecting the central nervous system (CNS). Environmental factors have been suggested as possible factors affecting both disease risk and progression in individuals with complex genetic-risk profiles [2].

MS is not yet curable and therapeutic options mainly focus on the control of the autoimmune reaction. The approved therapies include immunomodulatory (interferon-beta (IFN- $\beta$ ), glatiramer acetate) and immunosuppressive agents (fingolimod, azathioprine, mitoxantrone) or antibody mediated (natalizumab) approaches [3]. Although efficacy of all these drugs has been proven in large-scale clinical trials, some major issues still remain unsolved including partial efficacy, considerable variety inpatients response, inconvenience of parenteral application, safety and tolerability aspects, insufficient neuroprotective capacity, and last but not least considerable treatment costs [3]. Against this background the need for an improvement of therapeutic options in MS remains. Identifying therapies that have the potential to alter the disease course with a low side-effect profile are needed.

\section{Complementary and Alternative Medicine Use in MS}

There have been a number of studies reporting the prevalence of complementary and alternative medicine (CAM) use by MS patients, and the range of prevalence is quite broad, at 33-70\% [4-8]. The majority of MS patients uses CAM as an adjunct to conventional therapies and perceives benefits from both therapies. CAM therapies most frequently employed include diets, $\omega-3$ polyunsaturated fatty acids (PUFAs), antioxidants [9-12].

The role of diet in both causing and ameliorating the severity of MS has intrigued people with MS and researchers alike. One of the first researchers to suggest a relationship between dietary habits and MS was Swank who conducted epidemiological studies of MS in Norway
[13]. He found a lower incidence in the coastal communities with high consumption of fish (rich in $\omega-3$ PUFAs) than in communities where the consumption of animal fat is high. He concluded that a high butterfat intake leads to an increased risk of developing MS, whereas a fish-based diet is associated with a decreased risk of MS. He also developed dietary recommendations based on these findings that were widely used by MS patients before the introduction of diseasemodifying therapies in the mid-to-late 1990s.

\section{$\omega$-3 Polyunsaturated Fatty Acids (PUFAs) and Immune Response}

$\omega-3$ fatty acids are a range of dietary PUFAs characterized by having a $\mathrm{C}=\mathrm{C}$ bond starting after the third carbon atom from the methyl end of the carbon chain. The predominant $\omega-3$ fatty acids are $\alpha$-linolenic acid (ALA; 18:3 $\omega$-3), eicosapentaenoic acid (EPA; 20:5 $\omega$ -

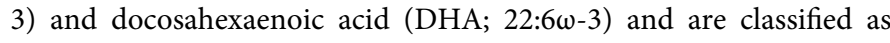
essential fatty acids in humans because they are a requirement for the maintenance of health and cannot be synthesized de novo, although limited conversion of ALA to the longer chain fatty acids, EPA and DHA can occur, particularly in men [14,15]. Algae are the primary natural source of EPA and DHA, and as a result of their consumption and dietary accumulation, fish are a rich source of these fatty acids.

Both human and animal studies have proved that diets high in EPA and DHA can increase the proportion of these PUFAs in cell membranes and also reduce the levels of arachidonic acid (AA, C20:4 $\omega-6$ ), a stressrelated biomarker [16].EPA and DHA are able to inhibit partly a number of aspects of inflammation including leucocyte chemotaxis, adhesion molecule expression and leucocyte-endothelial adhesive interactions,

*Corresponding author: Serafina Salvati, Department Veterinary Public Health \& Food Safety, IstitutoSuperiore di Sanità, Rome, Italy, Tel: +39 06 49902574; Fax: +39 06 49387149; E-mail: serafina.salvati@iss.it

Received December 26, 2013; Accepted February 19, 2014; Published February 27,2014

Citation: Salvati S, Di Biase A (2014) $\omega-3$ Polyunsaturated Fatty Acids on the Prognosis of Multiple Sclerosis: The Effect of Eicosapentaenoic acid. J Neurol Neurophysiol S12: 009. doi:10.4172/2155-9562.S12-009

Copyright: ( 2014 Salvati S, et al. This is an open-access article distributed under the terms of the Creative Commons Attribution License, which permits unrestricted use, distribution, and reproduction in any medium, provided the original author and source are credited. 
production of inflammatory cytokines and T cell reactivity. These $\omega-3$ PUFAs function through a variety of mechanisms, including via cell surface (GPR120) and intracellular NR1C3 (i.e. PPAR- $\gamma$ ) receptors that control inflammatory cell signaling and gene expression patterns [17]. In addition, $\omega$-3 PUFAs can modify the production of lipid mediators. This occurs through several mechanisms that are all relate to the fatty acid composition of cell membrane phospholipids: 1) the decreased amount of AA results in decreased production of eicosanoids like serie-2 prostaglandins (PGs) and 4-series leukotrienes (LTs) from inflammatory cells;2) EPA acts as an alternative substrate for cyclooxygenase (COX) and lipoxygenases (LOX) enzymes, giving rise to 3 -series PGs and tromboxanes (TXs) and 5-series LTs. The difference between $\omega-3$ and $\omega-6$ fatty acid-derived eicosanoids is that most of the mediators formed from EPA and DHA are anti-inflammatory, whereas those formed from AA are pro-inflammatory or show other diseasepropagating effects [18]. Furthermore, EPA and DHA also give rise to resolvins (from EPA and DHA) and neuroprotectins (from DHA) through pathways involving COX and LOX enzymes [19,20]. Among $\omega-3$ PUFAs, EPA appears to have the most potent effects on immune response [21,22]. Moreover, recent studies provided evidence that one effect of EPA may involve induction of CD4+CD25+ regulatory $\mathrm{T}$ cells (Tregs) [23] that appear to be critical in regulating immune response to self-antigens.

\section{w-3 PUFAs and Multiple Sclerosis}

The immune modulator and anti-inflammatory effects of $\omega-3$ PUFAs have been suggested as rationale for their potential therapeutic use in patients with MS.

Clinical trials related to $\omega$-3 PUFAs supplementation pointed out some benefits for RRMS. The largest study, with over 300 patients, used $10 \mathrm{~g}$ fish oil supplementation as the intervention and olive oil as placebo [24]. Although not statistically significant difference was observed, there was a trend favoring the $\omega$-3 PUFAs group, with fewer people progressing one point on the Expanded Disability Status Scale (EDSS). Beneficial trends on decreasing the duration and the frequency of MS relapses in favor of treatment group were also observed.

An open-label study [25] showed that $\omega$-3 PUFAs supplementation (6 g/day of fish oil; 86\% EPA+DHA) for 6 months in MS patients and healthy controls decreases the levels of interleukin-1ß (IL$1 ß)$, tumor necrosis factor- $\alpha$ (TNF- $\alpha$ ), IL-2, and IFN- $\beta$ produced from un stimulated and stimulated peripheral blood mononuclear cells (PBMC)as well as reduces the secretion of the inflammatory eicosanoids, prostaglandin E2 and leukotrienes LTB4, which are known to be increased in MS patients [26] Weinstock-Guttman et al. [27] investigated fish oil and other dietary fat changes as a treatment among users of approved MS conventional therapies. In a randomized, double-blind, placebo-controlled study a significant improvement in quality of life (physical and mental health components) was observed in participants receiving a low saturated fat diet and $\omega-3$ PUFAs supplementation ( $6 \mathrm{~g}$ /day of fish oil; $65 \% \mathrm{EPA}+\mathrm{DHA})$ at 6 months (Table 1). In this study no changes in plasma inflammatory cytokine levels were observed. The difference in outcomes on inflammatory cytokine levels between Weistock-Guttman et al. and Gallai et al. studies can be due to the different concentrations of EPA and DHA evaluated (Weistock-Guttman et al. evaluated a lower concentration than Gallai et al.) and different specimen used in cytokine measures (plasma versus immune cell secreted). Despite these differences, both studies reported positive effects of $\omega-3$ PUFA supplementation in MS. However, the potential therapeutic effect related to a low fat diet cannot be clearly excluded in this last study.

In a multicenter, randomized, placebo-controlled trial, in which 92 patients with RRMS received either fish oil (5 g/day; 60\% EPA +DHA) or placebo, no significant difference was found in either the number of lesions on gadolinium-enhanced magnetic resonance imaging (MRI) at 6 months or the relapse rate at 6 or 24 months [28]. Furthermore, no differences were detected in disability progression, fatigue or qualityof-life scores. However, no dietary restrictions or recommendations were given to participants, which might have attenuated any benefit of the fatty acid supplementation.

Swank and Goodwin [29] conducted a 35-year long, nonrandomized, retrospective study in MS patients and found that a diet with very low saturated fat content (average saturated fat 15-17 g/day) supplemented with cod liver or vegetable oils (10-40 g/day) provided long term benefits on mortality, relapse severity and disability, particularly if initiated during the earliest stages of MS. Although this study is a unique long-term follow-up of an intervention for MS, it has been criticized for the lack of a control group for comparison for scientific validation.

Recently, Pantzaris et al. [30] showed in a randomized, doubleblind, placebo-controlled clinical trial that the administration of $\omega-3$ (EPA $1.65 \mathrm{~g} /$ day and DHA $4.65 \mathrm{~g} /$ day) and $\omega-6$ fatty acids with vitamins reduce the annualized relapse rate in RRMS. However, in this study it is not possible to discriminate the effects of $\omega-3$ and $\omega-6$ fatty acids.

The available data are insufficient to assess the real effect of these compounds in MS. In addition high dropout rates limit the conclusions that can be drawn from these studies. Given positive trends observed in small studies, the potential of $\omega$-3 PUFAs to slow the disease course should be evaluated further. Among $\omega-3$ PUFAs, EPA has the most potent effect on inflammatory processes $[31,32]$ and the conflicting

\begin{tabular}{|c|c|c|c|c|c|}
\hline Author & Patients & Type of study & Intervention & $\mathbf{n}$ & Main results \\
\hline Bates (1989) [24] & RRMS & $\begin{array}{l}\text { RCT, double blind parallel } \\
\text { design; }\end{array}$ & $\begin{array}{l}\text { EPA } 1.71 / \mathrm{DHA} 1.14 \mathrm{~g} / \text { day vs. Oleic acid } \\
7.2 \mathrm{~g} / \text { day }\end{array}$ & $\begin{array}{l}145 \\
147\end{array}$ & $\begin{array}{l}\text { No Significant effect on relapse rate and } \\
\text { disability progression. Positive trend } \\
\text { In fish oil group }\end{array}$ \\
\hline $\begin{array}{l}\text { Weinstock-Guttmann } \\
\text { (2005) [27] }\end{array}$ & RRMS & $\begin{array}{l}\text { RCT, double blind parallel } \\
\text { design; } 12 \text { month follow-up. } \\
\text { IMT allowed }\end{array}$ & $\begin{array}{l}\text { EPA 1.98/DHA } 1.32 \mathrm{~g} / \text { day+dietary fat } \\
\text { restriction } \\
\text { Vs. } \\
\text { Olive oil } 6 \mathrm{~g} / \text { day+dietary fat restriction }\end{array}$ & $\begin{array}{l}13 \\
14\end{array}$ & $\begin{array}{l}\text { No effect on relapse rate or disability. } \\
\text { Positive effect } \\
\text { on mental and physical health related to } \\
\text { quality of life in fish oil group }\end{array}$ \\
\hline Torkildsen (2012) [28] & RRMS & $\begin{array}{l}\mathrm{RCT} \text {, double blind parallel } \\
\text { design; } 24 \text { month follow-up }\end{array}$ & $\begin{array}{l}\text { EPA 1.35/DHA } 0.85 \text { g/day vs. Corn oil } 5 \\
\text { g/day }\end{array}$ & $\begin{array}{l}46 \\
46\end{array}$ & $\begin{array}{l}\text { No effect of } \omega \text {-3 PUFAs on MRI-activity, } \\
\text { relapse rate or disability progression neither } \\
\text { as monotherapy nor as add-on to IFN- } \beta\end{array}$ \\
\hline Pantzaris (2013) [30] & RRMS & $\begin{array}{l}\mathrm{RCT} \text {, double blind parallel } \\
\text { design; } 30 \text { month follow-up }\end{array}$ & $\begin{array}{l}\text { EPA 1.65/DHA } 4,65 \mathrm{~g} / \text { day GLA } 2.0 / \mathrm{LA} \\
3.85 \mathrm{~g} / \text { day+vit A, vit E, } \mathrm{y} \text {-tocopherol }(0.6 \text {, } \\
22 \text {, and } 760 \mathrm{mg} / \text { day) vs. Olive oil } 16.9 \mathrm{~g} / \\
\text { day }\end{array}$ & $\begin{array}{l}20 \\
20\end{array}$ & $\begin{array}{l}\text { Significant reduction of the annual relapse } \\
\text { rate and disability progression }\end{array}$ \\
\hline
\end{tabular}

Table 1: Randomized controlled trials of $\omega-3$ PUFAs in MS. 
results obtained in different clinical trials could be due to the fact that fish oils from different sources contain variable mixtures of EPA and DHA.

\section{EPA and Myelin Gene Expression}

In addition to having anti-inflammatory properties, $\omega$-3 PUFAs have proved to have beneficial effects on myelinogenesis. In an in vitro study, Van Meeteren showed that the supplementation of oligodendroglia cells with $\omega$-3 PUFAs increased their degree of differentiation [33]. In our in vitro experimental model using transfected C6 glioma cells with proteolipid protein (PLP) promoter [34], we showed that EPA stimulates PLP gene expression via cAMP mediated pathways. Afterwards, to verify whether the $\omega$-3 PUFAs have an in vivo role on the myelinogenesis process, a single dose of EPA or DHA was injected into brains of rat pups, and myelin development was assessed 3 days later by evaluating myelin protein expression in medulla, cerebellum, and cortex. Our results showed that the levels of PLP, myelin basic protein, and myelin oligodendrocyte protein mRNAs increased in nearly all brain regions of DHA and EPA treated animals, but the effect was more pronounced in EPA treated rats. The enhancement in PLP transcript levels was followed by an increase in PLP translation in EPA treated rats. A further indicator of accelerated myelination was the increase in 2-3-cyclic nucleotide 3- phosphodiesterase (myelin marker) protein levels [35].

In MS treatment, the enhancement of endogenous remyelination represents an attractive therapeutic goal. Spontaneous and robust remyelination occurs at the early stages of MS. However, remyelination is incomplete - i.e., faithful reconstruction is not fully attained, originating a thinner and shorter myelin sheath. As the disease progresses, eventually remyelination completely fails. The reasons for this inadequacy are unknown, as the oligodendrocyte precursor cells (OPCs), the cell population that is considered to be the most important source of remyelinating oligodendrocytes in the adult CNS, are present in early demyelinating lesions in MS.

There are many possible speculative explanations for remyelination failure in MS, including quantitatively inadequate recruitment and/ or differentiation of OPCs; axons not receptive to remyelination; and inappropriate support of growth factors by astrocytes and/or other inflammatory cells, such as the extracellular microenvironment with regard to matrix proteins and adhesion molecules [36]. Thus, extensive studies are under way to identify factors involved in OPC differentiation during remyelination. It is generally accepted that the process of remyelination represents a recapitulation of myelination during development, and so the key factors affecting the developmental maturation of OPCs into myelinating oligodendrocytes also should favor remyelination in the adult CNS.

Thus, in addition to $\omega$-3 PUFA effect on decreasing proinflammatory cytokines levels, there is evidence supporting $\omega-3$ PUFAs effect in myelin genes both in vitro and in vivo and EPA has the major effect.

\section{EPA and Experimental Allergic Encephalomyelitis}

The effects of EPA were investigated in EAE induced in dark agouti (DA)rat which develops a severe and protracted encephalitis and wide demyelination $[37,38]$. In the DA rat EAE model, following the first attack and remission, animals experience pathology similar to MS patients with T cells, macrophages, glial cells and, additionally, autoantibody-producing plasma cells involving in demyelination axonal loss spreading beyond the spinal cord into the cerebellum. Most of the animals also relapse; thus the similarity of disease in the
DA rat EAE model to the human disease renders this strain a valid and useful model for testing potential MS therapies. We observed that prophylactic administration of EPA conferred significant protection against development of EAE [39]. The protective role was manifested at clinical, histological and molecular levels. In particular, we observed a delayed onset of clinical severity of disease in rats fed EPAsupplemented diets that were associated to an increased expression of myelin proteins and an improved integrity of the myelin sheath. The increased expression of NG2-chondroitin sulfate proteoglycan (OPCs marker) and PLP protein levels observed in treated rats suggests that EPA improves myelin self-repair capability by unblocking OPCs and pushing them toward becoming mature myelinating OLs. Thus EPA seems to reach a major goal of MS therapy: to overcome the remyelination failure.

Moreover, an up-regulation of expression of fork head transcription factor (Foxp3, the most specific marker for Treg cells) in the CNS of EPA feeding rats during the acute phase of EAE was observed. Tregs are capable of controlling the development and activity of pathogenic effector T cells during autoimmune diseases through cell-to-cell contact or cytokine-dependent pathways, respectively [40]. Defective Treg function may be important in the pathogenesis of MS as well as in its rodent models [41,42]. For example, targeted deletion of Tregs causes spontaneous autoimmune disease in mice, whereas augmentation of Treg function can prevent the development or alleviate variants of EAE [43].

Based on evidences that EPA could have beneficial and protective effects directly on oligodendrocytes, we have evaluated the effects of EPA in the MS model characterized by toxic demyelination induced by cuprizone (CPZ). CPZ feeding, with mechanisms not yet well established, induces oligodendrocyte degeneration, disruption of myelin sheath and demyelination mainly localized in the medial corpus callosus (CC) [44]. In our study, EPA was daily administered by gavage to newborn rats for 21 days, and then they were fed for 9 days on the $\mathrm{CPZ}$ diet. The results showed that EPA was able to partially protect the brain against damage induced by $\mathrm{CPZ}$ intoxication [45].

\section{Conclusion}

In summary, EPA may have a potential benefit in MS for its immunomodulatory and neuroprotective properties. In fact, pathological demyelination and the corresponding repair mechanism requires a finely orchestrated interplay between the inflammatory immune cells that mediate the damage but also stimulate myelin repair by oligodendrocyte precursor cells. Evidence on the possible benefits of EPA in MS is lacking. Previous published trials were focused on the effect of fish oil on MS. The conflicting results reported in literature could be due to the different mixture of EPA and DHA present in the different sources of fish oil so that the EPA amount could be too low to exert a therapeutic action. Further studies should be designed to evaluate the effectiveness of EPA both as monotherapy and in combination with disease-modifying drugs in MS.

\section{References}

1. Hassan-Smith G, Douglas MR (2011) Epidemiology and diagnosis of multiple sclerosis. Br J Hosp Med (Lond) 72: M146-151.

2. Compston A, Coles A (2008) Multiple sclerosis. Lancet 372: 1502-1517.

3. Río J, Comabella M, Montalban X (2011) Multiple sclerosis: current treatment algorithms. Curr Opin Neurol 24: 230-237.

4. Yadav V, Shinto L, Bourdette D (2010) Complementary and alternative medicine for the treatment of multiple sclerosis. Expert Rev Clin Immunol 6 : 381-395. 
Citation: Salvati S, Di Biase A (2014) $\omega-3$ Polyunsaturated Fatty Acids on the Prognosis of Multiple Sclerosis: The Effect of Eicosapentaenoic acid. J Neurol Neurophysiol S12: 009. doi:10.4172/2155-9562.S12-009

5. Leong EM, Semple SJ, Angley M, Siebert W, Petkov J, et al. (2009) Complementary and alternative medicines and dietary interventions in multiple sclerosis: what is being used in South Australia and why? Complement Ther Med 17: 216-223.

6. Marrie RA, Hadjimichael O, Vollmer T (2003) Predictors of alternative medicine use by multiple sclerosis patients. Mult Scler 9: 461-466.

7. Schwarz S, Knorr C, Geiger H, Flachenecker P (2008) Complementary and alternative medicine for multiple sclerosis. Mult Scler 14: 1113-1119.

8. Shinto L, Calabrese C, Morris C, Yadav V, Griffith D, et al. (2008) A randomized pilot study of naturopathic medicine in multiple sclerosis. J Altern Complement Med 14: 489-496.

9. Cunningham E (2013) Are there evidence-based dietary interventions for multiple sclerosis? J Acad Nutr Diet 113: 1004

10. Rezapour-Firouzi S, Arefhosseini SR, Mehdi F, Mehrangiz EM, Baradaran B et al. (2013) Immunomodulatory and therapeutic effects of Hot-nature diet and co-supplemented hemp seed, evening primrose oils intervention in multiple sclerosis patients. Complement Ther Med 21: 473-480.

11. Maker-Clark G, Patel S (2013) Integrative therapies for multiple sclerosis. Dis Mon 59: 290-301.

12. von Geldern G, Mowry EM (2012) The influence of nutritional factors on the prognosis of multiple sclerosis. Nat Rev Neurol 8: 678-689.

13. SWANK RL, LERSTAD O, STRØM A, BACKER J (1952) Multiple sclerosis in rural Norway its geographic and occupational incidence in relation to nutrition. N Engl J Med 246: 722-728.

14. Fetterman JW Jr, Zdanowicz MM (2009) Therapeutic potential of n-3 polyunsaturated fatty acids in disease. Am J Health Syst Pharm 66: 1169-1179.

15. Lands WE (1992) Biochemistry and physiology of $n-3$ fatty acids. FASEB J 6: 2530-2536.

16. Calder PC (2006) n-3 polyunsaturated fatty acids, inflammation, and inflammatory diseases. Am J Clin Nutr 83: 1505S-1519S.

17. Simopoulos AP, Leaf A, Salem N (1999) Workshop on the essentiality of and recommended dietary intakes for omega- 6 and omega- 3 fatty acids. J Am CollNutr 18: 487-489.

18. Burdge GC, Calder PC (2005) Conversion of alpha-linolenic acid to longerchain polyunsaturated fatty acids in human adults. Reprod Nutr Dev 45: 581 597.

19. Calder PC (2013) Omega-3 polyunsaturated fatty acids and inflammatory processes: nutrition or pharmacology? Br J Clin Pharmacol 75: 645-662.

20. Yates CM, Calder PC, Ed Rainger G (2014) Pharmacology and therapeutics of omega-3 polyunsaturated fatty acids in chronic inflammatory disease. Pharmacol Ther 141: 272-282.

21. Calder PC (1996) Sir David Cuthbertson Medal Lecture. Immunomodulatory and anti-inflammatory effects of $\mathrm{n}-3$ polyunsaturated fatty acids. Proc Nutr Soc 55: 737-774.

22. Gorjão R, Azevedo-Martins AK, Rodrigues HG, Abdulkader F, Arcisio-Miranda $M$, et al. (2009) Comparative effects of DHA and EPA on cell function. Pharmacol Ther 122: 56-64.

23. Iwami D, Nonomura K, Shirasugi N, Niimi M (2011) Immunomodulatory effects of eicosapentaenoic acid through induction of regulatory $\mathrm{T}$ cells. Int Immunopharmacol 11: 384-389.

24. Bates D, Cartlidge NE, French JM, Jackson MJ, Nightingale S, et al. (1989) A double-blind controlled trial of long chain $n-3$ polyunsaturated fatty acids in the treatment of multiple sclerosis. J Neurol Neurosurg Psychiatry 52: 18-22.

25. Gallai V, Sarchielli P, Trequattrini A, Franceschini M, Floridi A, et al. (1995) Cytokine secretion and eicosanoid production in the peripheral blood mononuclear cells of MS patients undergoing dietary supplementation with $n-3$ polyunsaturated fatty acids. J Neuroimmunol. 56: 143-153.

26. Neu I, Mallinger J, Wildfeuer A, Mehlber L (1992) Leukotrienes in the cerebrospinal fluid of multiple sclerosis patients. Acta Neurol Scand 86: 586587.

27. Weinstock-Guttman B, Baier M, Park Y, Feichter J, Lee-Kwen P, et al. (2005) Low fat dietary intervention with omega-3 fatty acid supplementation in multiple sclerosis patients. Prostaglandins Leukot Essent Fatty Acids 73: 397-404.
28. Wergeland S, Torkildsen $\varnothing, B \varnothing \mathrm{L}$, Myhr KM (2012) Polyunsaturated fatty acids in multiple sclerosis therapy. Acta Neurol Scand Suppl 70-75.

29. Swank RL, Goodwin J (2003) Review of MS patient survival on a Swank low saturated fat diet. Nutrition 19: 161-162.

30. Pantzaris MC, Loukaides GN, Ntzani EE, Patrikios IS (2013) A novel ora nutraceutical formula of omega-3 and omega- 6 fatty acids with vitamins (PLP 10 ) in relapsing-remitting multiple sclerosis: a randomised, double-blind, placebo-controlled proof-of-concept clinical trial. BMJ Open 3: 1-16.

31. Sierra S, Lara-Villoslada F, Comalada M, Olivares M, Xaus J (2008) Dietary eicosapentaenoic acid and docosahexaenoic acid equally incorporate as decosahexaenoic acid but differ in inflammatory effects. Nutrition 24: 245-254.

32. Verlengia R, Gorjão R, Kanunfre CC, Bordin S, Martins De Lima T, et al. (2004) Comparative effects of eicosapentaenoic acid and docosahexaenoic acid on proliferation, cytokine production, and pleiotropic gene expression in Jurkat cells. J Nutr Biochem 15: 657-665.

33. van Meeteren ME, Baron W, Beermann C, Dijkstra CD, van Tol EA (2006) Polyunsaturated fatty acid supplementation stimulates differentiation of oligodendroglia cells. Dev Neurosci 28: 196-208.

34. Salvati S, Natali F, Attorri L, Raggi C, Di Biase A, et al. (2004) Stimulation of myelin proteolipid protein gene expression by eicosapentaenoic acid in $\mathrm{C} 6$ glioma cells. Neurochem Int 44: 331-338.

35. Salvati S, Natali F, Attorri L, Di Benedetto R, Leonardi F, et al. (2008) Eicosapentaenoic acid stimulates the expression of myelin proteins in rat brain. J Neurosci Res 86: 776-784.

36. Franklin RJ, ffrench-Constant C, Edgar JM, Smith KJ (2012) Neuroprotection and repair in multiple sclerosis. Nat Rev Neurol 8: 624-634.

37. Swanborg RH (1995) Experimental autoimmune encephalomyelitis in rodents as a model for human demyelinating disease. Clin Immunol Immunopathol 77 : 4-13.

38. Lorentzen JC, Issazadeh $\mathrm{S}$, Storch $\mathrm{M}$, Mustafa MI, Lassman $\mathrm{H}$, et al (1995)Protracted, relapsing and demyelinating experimental autoimmune encephalomyelitis in DA rats immunized with syngeneic spinal cord and incomplete Freund's adjuvant. J Neuroimmunol 63: 193-205.

39. Salvati S, Di Biase A, Attorri L, Di Benedetto R, Sanchez M, et al. (2013) Ethyleicosapentaenoic acid ameliorates the clinical course of experimental allergic encephalomyelitis induced in dark agouti rats. J Nutr Biochem 24: 1645-1654.

40. Costantino CM, Baecher-Allan CM, Hafler DA (2008) Human regulatory T cells and autoimmunity. Eur J Immunol 38: 921-924.

41. Zozulya AL, Wiendl $\mathrm{H}$ (2008) The role of regulatory $\mathrm{T}$ cells in multiple sclerosis. Nat Clin Pract Neurol 4: 384-398.

42. Matsumoto Y, Sakuma H, Kohyama K, Park IK (2007) Paralysis of CD4(+) $\mathrm{CD} 25(+)$ regulatory $\mathrm{T}$ cell response in chronic autoimmune encephalomyelitis. J Neuroimmunol 187: 44-54.

43. Aharoni R, Eilam R, Stock A, Vainshtein A, Shezen E, et al. (2010) Glatiramer acetate reduces Th-17 inflammation and induces regulatory T-cells in the CNS of mice with relapsing-remitting or chronic EAE. J Neuroimmunol 225: 100-111.

44. Matsushima GK, Morell P (2001) The neurotoxicant, cuprizone, as a model to study demyelination and remyelination in the central nervous system. Brain Pathol 11: 107-116.

45. Di Biase A, Salvati S, Di Benedetto R, Attorri L, Martinelli A, et al. (2013) Eicosapentaenoic acid pre-treatment reduces biochemical changes induced in total brain and myelin of weanling Wistar rats by cuprizone feeding Prostaglandins LeukotEssent FattyAcidsDec. 7 DOI: 10.1016/j.plefa 2013.11.004

This article was originally published in a special issue, Neurodegenerative Diseases: Symptoms and Therapeutics handled by Editor(s). Dr. Jin J Luo, Temple University School of Medicine, USA 